\title{
Sind IT-gestützte klinische Behandlungspfade der Papierform überlegen?
}

\author{
Are IT-Based Clinical Pathways Superior to Hard-Copy Form?
}

Autoren

Institute
L. Homagk' ${ }^{1}$ I. Wiesner ${ }^{2}$, G. O. Hofmann ${ }^{3}$, J. Zaage ${ }^{2}$

BG-Kliniken Bergmannstrost, Klinik für Unfall- und Wiederherstellungschirurgie, Halle, Deutschland

BG Kliniken Bergmannstrost, Klinik für Allgemein- und Viszeralchirurgie, Halle, Deutschland

Friedrich-Schiller-Universität Jena, Klinik für Unfall-, Hand- und Wiederherstellungschirurgie, Jena, Deutschland
Schlüsselwörter

- Behandlungspfad

- Planungsbogen

D laparoskopische Chirurgie

Key words

- clinical pathway

- electronic file

- laparoscopic surgery

\section{Bibliografie}

DOI http://dx.doi.org/ 10.1055/s-0030-1262608

Online-Publikation 2.5.2011

Zentralbl Chir 2013; 138: 64-69

(c) Georg Thieme Verlag KG

Stuttgart . New York .

ISSN 0044-409X

\section{Korrespondenzadresse}

Dr. Lars Homagk

BG Kliniken Bergmannstrost .

Unfall- und Wiederherstellungschirurgie

Merseburgerstr. 165

06112 Halle

Deutschland

Tel.: $0345 / 1327827$

Fax: 0345/1326497

laho@gmx.net

\section{Zusammenfassung \\ $\nabla$}

Hintergrund: Klinische Behandlungspfade („clinical pathways“) sind heute in vielen Kliniken, analog zum industriellen Prozessmanagement, zur strukturierten Ablauforganisation in der chirurgischen, interventionellen sowie konservativen Behandlung etabliert.

Fragestellung: In unserer Arbeit wurde die Umstellung von papiergebundenen Behandlungspfaden zur IT-gestützten Form mit der Frage einer Qualitätsänderung der Behandlungsabläufe evaluiert.

Methodik: In unserer Klinik für Allgemein- und Viszeralchirurgie wurden 4 bestehende Behandlungspfade sowie 1 neuer Pfad als IT-gestütztes Routinearbeitsinstrument in unserem Krankenhausinformationssystem (KIS) implementiert. Es erfolgte ein Abgleich der präoperativen und stationären Behandlungsdauer und des Schmerzempfindens.

Ergebnisse: In der Allgemeinchirurgie wurden bis Juli 2009 die papiergebundenen Behandlungspfade der laparoskopischen Cholezystektomie, der Hernioplastik, Strumektomie und des Varizenstrippings in das KIS implementiert sowie der Pfad Kolonresektion neu entwickelt. Vom 1.1.2006 bis zum 31.10.2009 wurden in den 2 ausgewerteten Behandlungspfaden der laparoskopischen Cholezystektomie und Hernioplastik 743 Patienten erfasst. Davon wurden ab Juli 2009 51 Fälle nach IT-Pfad geführt. Dabei zeigte sich bei der Hernioplastik eine Reduktion der präoperativen Tage mit dem IT-Pfad, die weiteren untersuchten Parameter der Liegedauer und des subjektiven Schmerzempfindens waren ohne Unterschied.

Schlussfolgerung: Ein IT-gestützter klinischer Behandlungspfad ist als Routinearbeitsinstrument im KIS als kontinuierliche Fortsetzung klinischer papiergebundener Behandlungspfade einsetzbar und kann für einzelne operative Prozeduren als

\section{Abstract \\ $\nabla$}

Background: Today clinical pathways are established as a basis for the operational and organisational structure of surgical, interventional and conservative treatments in many hospitals.

Question: In our study we have evaluated the establishment and systematic applicability of ITbased clinical pathways in the department of general surgery in comparison with existing clinical pathways. Does the systematic application of IT-based clinical pathways improve the quality of medical treatment?

Methods: In our department of general surgery we evaluated and compared the treatment by laparoscopic surgery before and after establishment of IT-based clinical pathways. The preoperative duration of stay, the duration of treatment and the patients' pain perception were compared. Since July 2009 we used in our department of general surgery an IT-based clinical pathway for laparoscopic cholecystectomy and hernia repair. From January 2006 until October 2009 we compared the treatment by these procedures with and without the use of IT-based clinical pathways.

Results: From January 2006 until October 2009 743 patients underwent surgery and treatment following the conventional clinical pathway and 51 patients following the new, IT-based clinical pathway. In the group of patients who received a laparoscopic hernia repair we found a reduction of the preoperative duration of stay.

Conclusion: IT-based clinical pathways are applicable for routine use in general surgery departments. For certain surgical procedures they are an eligible management device. IT-based clinical pathways lead to an improved operational structure of medical treatment and moreover to a complete and continuous documentation through the electronic file. Especially for departments which are using the electronic file, the use of ITbased clinical pathways can be recommended. 
umfassendes klinisch-administratives Steuerungsinstrument angewandt werden. Dabei ist insbesondere in Häusern mit einem IT-gestützten KIS die Implementierung elektronischer Behandlungspfade zu empfehlen, da somit eine lückenlose elektronische Dokumentation im Rahmen der elektronischen Fieberkurve gewährleistet wird.

Klinische Behandlungspfade, häufig auch Planungsbögen oder Clinical Pathways genannt, werden schon seit vielen Jahren und steigender Frequenz zur strukturierten Ablauforganisation in der operativen, konventionellen und interventionellen Therapie in deutschen Krankenhäusern eingesetzt [1-4]. Dabei stellen sie den optimalen Ablauf einer definierten Behandlung der betreffenden Patienten in der ökonomisch sinnvollsten Reihenfolge dar und führen zur Verbesserung der Patientenzufriedenheit [5-7]. Behandlungspfade beschreiben dabei für alle beteiligten Berufsgruppen, wie ärztlicher, pflegerischer und Sozialdienst oder Krankengymnastik detailliert die erforderlichen diagnostischen und therapeutischen Prozeduren. Idealerweise durchläuft der Patient den Pfad im vorgegebenen Zeitrahmen seines stationären Aufenthalts. Dabei wird der Pfad den hohen Qualitätsanforderungen bei gleichzeitiger hoher ökonomischer Effizienz gerecht. Darüber hinaus fördern klinische Behandlungspfade Transparenz und Vergleichbarkeit einer Behandlung und können Behandlungsfehler minimieren [8]. Sie können die routinemäßige Dokumentation des normalen Behandlungsablaufs reduzieren und erlauben damit eine Zeitersparnis im Rahmen der Dokumentation [9]. Auf der Basis von Behandlungspfaden können zudem Abteilungsbudgets geplant werden. Sie dienen in der Weiterbildung als Leitfaden für junge Assistenzärzte, Patienten und Angehörige. Zusammenfassend sind die wichtigsten Ziele bei der Etablierung von Behandlungspfaden [10]:

- Transparente Ablauforganisation mit definierten Qualitätsund Behandlungszielen

- Verbesserung der Struktur-, Prozess-, und Ergebnisqualität als kumulierender Entwicklungsgang

- Verbesserung der Patientenzentrierung. -aufklärung und -zufriedenheit

- Sicherstellung der Ressourcenverfügbarkeit bei Vermeidung von überflüssigen Leistungen (Doppeluntersuchungen)

- Prozesskostentransparenz

- Evaluierung qualitativer und ökonomischer Ergebnisse mit der Möglichkeit der Prozessanpassung

- Optimierung der Falldokumentation und Konfliktvermeidung mit den Kostenträgern

Idealerweise erstreckt sich der Behandlungspfad von der Aufnahme bis zur Entlassung des Patienten und wird als berufsgruppen- und abteilungsübergreifendes Instrument geführt [11]. Aus dieser Analyse ergeben sich die geforderten Ein- und Ausschlusskriterien und mögliche Entscheidungskriterien für Alternativpfade. Im Weiteren erfolgen die Dokumentation des Ausgangszustands des Patienten und die Darstellung der Behandlungsund Qualitätsziele. Diese Maßnahmen werden im Rahmen der Ergebnisdokumentation mit dem Erreichen des gewünschten Behandlungszieles abgeschlossen. Die computergestützte Anwendung klinischer Behandlungspfade ermöglicht nun zusätzlich die Generierung von prozessierbaren Daten mit dem Ziel der Behandlungsoptimierung [6]. Darüber hinaus sind sie seit der Einführung der elektronischen Fieberkurve ein unausweichliches Arbeitsinstrument in der Fortführung der lückenlosen Behandlungsdokumentation.
Allerdings ist die wissenschaftliche Aufarbeitung des effizienten Einsatzes klinischer Behandlungspfade in Deutschland noch unzureichend. Wie erst kürzlich an gleicher Stelle berichtet wurde, beschäftigen sich ledig 3 Autoren intensiver mit dieser Problematik und eine prospektive Betrachtung konnte bislang nur einmal durchgeführt werden [12].

Zwar sind die ökonomischen und qualitativen Vorteile klinischer Pfade in diesen und ausländischen Studien mehrfach beschrieben worden, aber die Betrachtung elektronisch gestützter Behandlungspfade als Weiterentwicklung bereits bestehender papiergebundener Behandlungspfade ist noch nicht erfolgt.

Aus diesem Grund ist das Ziel dieser Arbeit die Beantwortung der Frage, ob die Umstellung der systematisch angewendeten papiergebundenen Behandlungspfade in IT-gestützte Behandlungspfade zu einer Qualitätsänderung der Behandlungsabläufe führt.

\section{Methoden}

$\nabla$

In unserem Klinikum der Schwerpunktversorgung werden im Jahresdurchschnitt ca. 13000 Patienten stationär behandelt. Ausgangspunkt für die Erstellung der Behandlungspfade war die standardisierte Behandlung häufiger allgemein- und viszeralchirurgischer Diagnosen und deren kostendeckende Abbildung im DRG-System. Dazu zählten in der Allgemeinchirurgie mit durchschnittlich 1100 stationären Patienten pro Jahr seit 2003 die laparoskopische Cholezystektomie, die Hernioplastik, die Strumektomie und das Varizenstripping sowie ab 2009 die Kolonresektion.

Wir entwickelten 2003 zunächst ein Kurvenblatt für die „Pfadgalle“, in dem mit deutlich reduziertem Aufwand alle perioperativen Daten dokumentiert werden konnten. Mit Einführung der elektronischen Fieberkurve ab 2006 in allen Kliniken unseres Hauses war es für uns erstrebenswert, die etablierten „PapierPfade" den neuen Entwicklungen anzupassen.

Die IT-Umsetzung wurde im klinikinternen KIS-System realisiert, welches auf jedem Rechner unserer Klinik installiert ist. Ein dezidiertes Berechtigungskonzept ermöglicht einen auf einzelne Berufsgruppen bezogenen, datenschutzrechtlich gesicherten $\mathrm{Zu}$ griff. Vor jedem Einsetzen der Behandlungspfade auf den Stationen wurden sie von den beteiligten Berufsgruppen auf ihre Praxistauglichkeit getestet und nach entsprechender Modifikation eingeführt.

Die Bearbeitung der klinischen Behandlungspfade beginnt, indem bei der stationären Aufnahme eines Patienten oder ambulanter Abklärung mit geplanter nachfolgender stationärer Aufnahme, ihm der jeweilige Pfad zugeordnet wird. Nach Eröffnen des Behandlungspfades ist auf einen Blick ersichtlich, welche Maßnahmen aktuell bereits bearbeitet sind und welche Maßnahmen noch offen sind oder zukünftig abzuarbeiten wären (o Abb. 1, 2).

Des Weiteren wird erkennbar, welche Berufsgruppe diese Tätigkeit zu erledigen hat. Neben den Hauptnutzern - ärztliche Mitarbeiter sowie Pflegekräfte - im Stations- und ambulanten Bereich, werden weitere Berufsgruppen oder Abteilungen in das vorhandene System eingebunden (Krankengymnasten, Sozialdienst, Röntgenabteilung und Anästhesie). Die abzuarbeitenden Behandlungsschritte sind jeweils mit den für diese Maßnahmen relevanten Informationen oder erforderlichen Formularen hinterlegt und können von den Zugangsberechtigten jederzeit abgerufen werden. Aktuell können derzeit aus dem Behandlungspfad heraus folgende Funktionalitäten genutzt werden ( 0 Abb.2): 


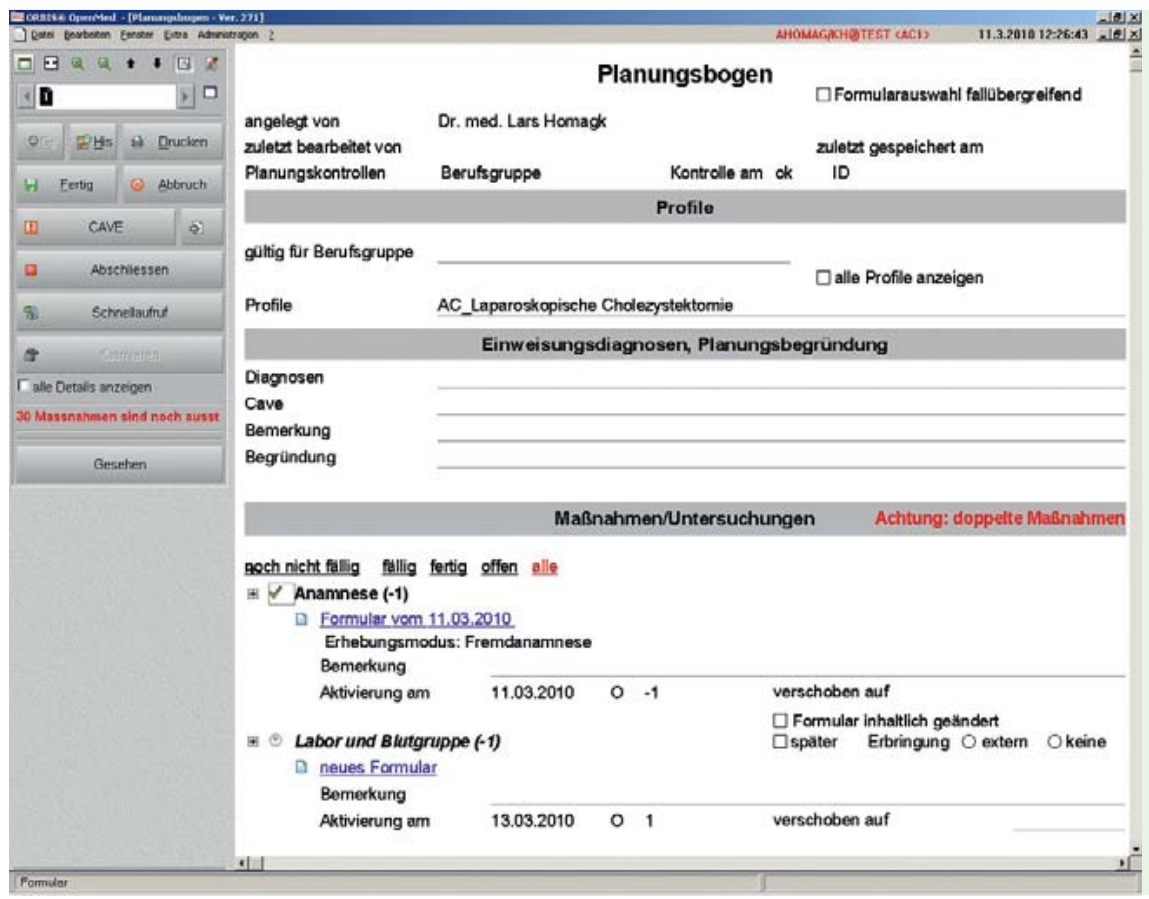

Abb. 1 Seite 1 des IT-Planungsbogens.

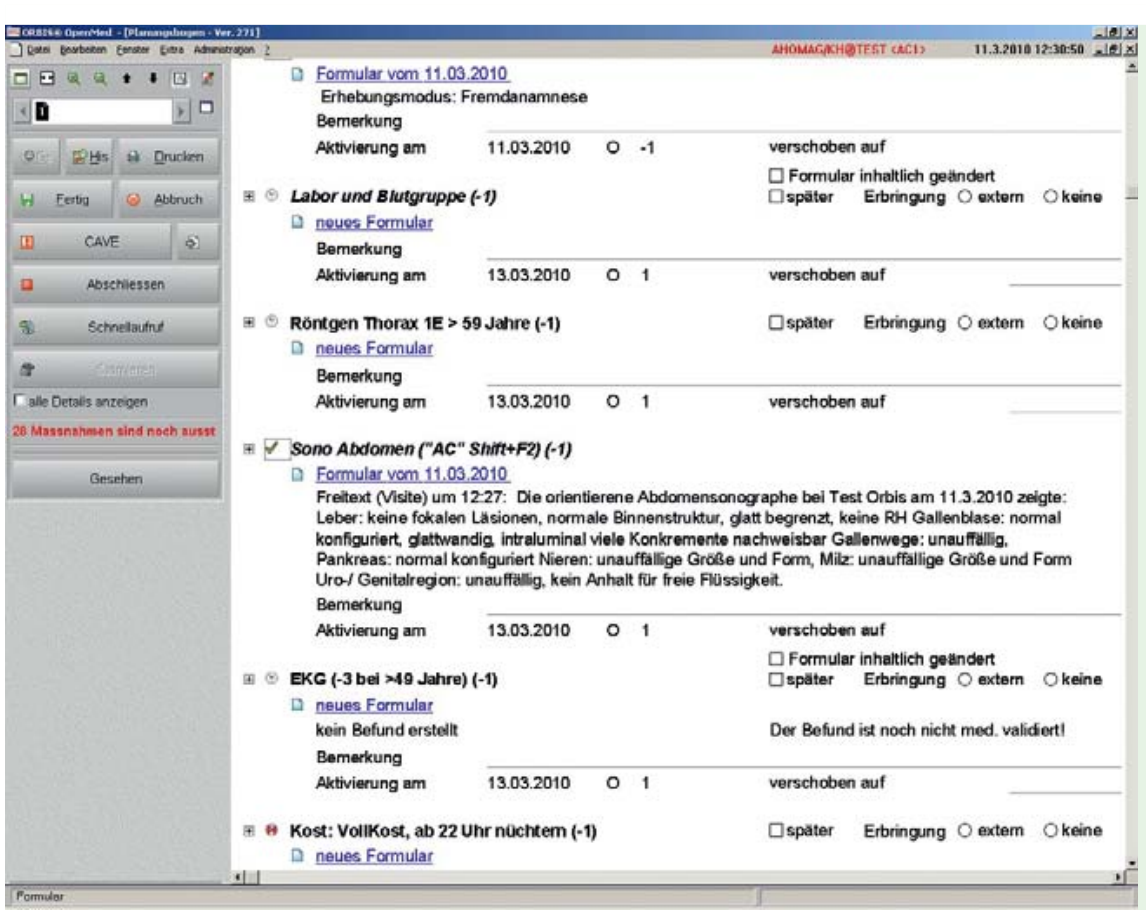

Abb. 2 Seite 2 des IT-Planungsbogens.

- Anamneseerstellung,

- automatische Erstellung sämtlicher Anforderungen (Röntgen, Labor, EKG, Krankengymnastik usw.),

- automatisches Erstellen und Weiterleiten erforderlicher Anordnungen (Konsile, Medikamente, Hilfsmittel, Essenbestellung etc.),

- Integration sämtlicher Befunde und Dokumentationen (Visite, Labor, Röntgen) in die elektronische Fieberkurve,

- automatische Erstellung des Entlassungsbriefes,

- Qualitätssicherungs-Dokumentation,

- tägliche Schmerzerfassung und ärztlicher Visitenbefund.

Beim Auslassen einer vorgegebenen Maßnahme kann in einem Freitextfeld die Begründung für diese „Pfadverletzung“ hinterlegt werden und zusätzlich definiert werden, ob diese Verletzung organisatorischer, medizinischer oder sonstiger Ursache war. Die Entscheidung eines Pfadabruchs obliegt dem federführenden Behandler.

Die Schmerzerfassung erfolgt in unserem Haus ebenfalls im Rahmen der elektronischen Fieberkurve anhand der Numerischen Ratingskala in der Einteilung $0=$ kein Schmerz bis $10=$ stärkster Schmerz.

\section{Ergebnisse}

Vom 1.1.2000 bis 31.12.2004 operierten wir in der Klinik für Allgemein- und Viszeralchirurgie 738 Patienten an der Gallenblase. Primär wurden in der Medizinischen Klinik 195(26,4\%) Patienten 
diagnostiziert und therapiert, wovon 184(75,1\%) im Rahmen des therapeutischen Splittings bei Verdacht auf Choledocholithiasis eine ERCP bzw. EPT erhielten. Nach Etablierung des Gallepfades am 1.8.2003 führten wir bis 13.9.2005 219 Cholezystektomien durch. Im Pfad abgebildet sind 168(76,1\%). Durch zusätzlich erforderliche präoperative Diagnostik und Therapie oder verlängerte postoperative Verweildauer waren $51(23,9 \%)$ nicht in den Ablaufplan der „Pfadgalle“ integrierbar. Dabei zeigt sich, dass präoperative Besonderheiten nur bei 3\% der Patienten zu Abweichungen führten. Bei 8,7\% war die Entlassung aus der stationären Behandlung erst 1 Tag später als vorgesehen möglich.

Vom 1.1.2006 bis zum 31.10.2009 wurden in 2 ausgewerteten Behandlungspfaden der laparoskopischen Cholezystektomie (lap. CCE) und der Hernioplastik (lap. HP) - 743 Patienten erfasst. Davon wurden ab Juli 200951 Fälle nach IT-Pfad geführt. Nach der Etablierung dieser Behandlungspfade in die IT-gestützte Form zeigte sich eine Verringerung der präoperativen Verweildauer bei der laparoskopischen Hernioplastik von 0,76 auf 0,35 Tage ( $\mathbf{A b b}$. 3). Die Gesamtzahl der nicht im Pfad integrierten Fälle bzw. Pfadabbrüche lag bei der laparoskopischen Hernioplastik bei 38,5\% und der laparoskopischen Cholezystektomie bei 20,0\% (D Tab. 1).

Weitere Unterschiede in den untersuchten Qualitäten der Gesamtliegedauer oder des subjektiven Schmerzempfindens bei der laparoskopischen Hernioplastik und „Pfadgalle“ ergaben sich nicht. Das subjektive Schmerzempfinden postoperativ lag zwischen 2,56 und 2,31 auf der Numerischen Ratingskala (O Abb.4).

\section{Diskussion}

Zusammenfassend können wir feststellen, dass die seit 2003 bestehende Durchführung der papiergebundenen Behandlungspfade in unserer Klinik für Allgemein- und Viszeralchirurgie eine hohe Akzeptanz unter dem pflegerischen und ärztlichen Personal gefunden hat und als Routineinstrument der Behandlungsführung etabliert ist. Daher haben wir auch in dieser Studie keine signifikante Verbesserung der Prozess- und Ergebnisqualität erwartet. Der große Nachteil und somit die Intention zur Umstellung auf einen IT-gestützten Pfad ist der Umstand der fehlenden Dokumentation in der elektronischen Krankenakte gewesen. Obwohl im Allgemeinen die Visiten mit einer elektronischen Bedside-Dokumentation des Befundes durchgeführt werden, ist bei den Pfadpatienten nur ein handschriftlicher Eintrag im Pfadblatt erfolgt. Somit fand nahezu keine Dokumentation im KIS statt, was insbesondere abrechnungstechnische Schwierigkeiten nach sich zog und mit Abschaffen der „Papierkurve“ in unserem Haus nicht mehr tragbar gewesen ist. Somit entschieden wir uns zur Umstellung der Behandlungspfade und wollten im Rahmen dieser Arbeit klären, ob diese Umstellung zu Qualitätsänderungen in der Behandlung geführt hat.

Die Studienlage zu klinischen Behandlungspfaden ist im deutschsprachigen Raum im Gegensatz zum angloamerikanischen Raum als spärlich zu bezeichnen. Die Ergebnis- und Prozessqualität

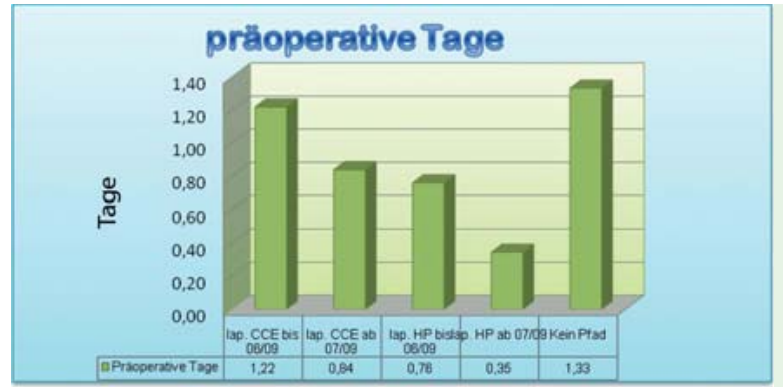

Abb.3 Darstellung der präoperativen Tage.

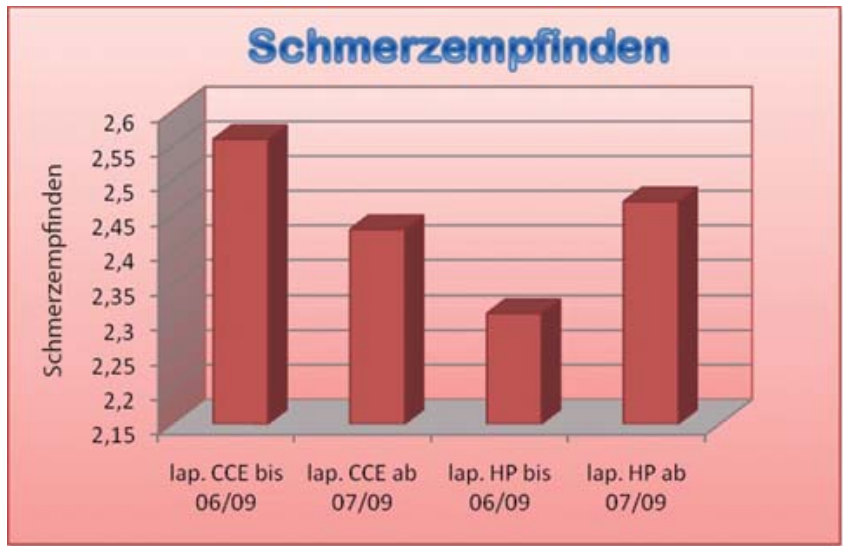

Abb.4 Darstellung des subjektiven Schmerzempfindens.

bleibt in der Literatur unterrepräsentiert [11]. Eine verminderte Behandlungsqualität durch Behandlungspfade ist bislang wissenschaftlich nicht belegt, da viele Studien hauptsächlich ökonomische Aspekte untersuchen, wobei sich eine Ersparnis durch den Einsatz von Behandlungspfaden sowohl für komplexe als auch für einfache Eingriffe zeigt [13-16]. Wir sehen es daher als erwiesen an, dass ein implementierter Behandlungspfad einen ökonomischen Benefit aufweist. Unser Schwerpunkt lag daher auf der Einführung IT-gestützter Behandlungspfade bei bereits bestehenden papiergebundenen Pfaden.

IT-gestützte Pfadanwendungen finden in der täglichen Routine eher eine zögerliche Akzeptanz, obwohl sie deutliche Vorteile hinsichtlich einer automatischen Erfassung behandlungsrelevanter Daten besitzen [17]. Unsere eigene Erfahrung dabei zeigte, dass ein hohes Maß an Zusammenarbeit und Verständnis für individuelle Aufgabenstellung zwischen der Softwareentwicklung und den ärztlichen Vorstellungen von Nöten ist. Die größten Probleme bei der elektronischen Umsetzung des Pfades waren in unserem Haus zu lange Wartezeiten beim Aufrufen der Pfade im Computer und anfängliche Kompatibilitätsprobleme externer Leistungsanforderungen, z.B. Röntgen, Labor etc. Inzwischen

\begin{tabular}{|lccccc|}
\hline Gruppe & $\begin{array}{l}\text { Summe } \\
\text { OPs }\end{array}$ & kein Pfad & Liegedauer & $\begin{array}{l}\text { präop. } \\
\text { Tage }\end{array}$ & $\begin{array}{l}\text { Schmerz- } \\
\text { empfinden }\end{array}$ \\
\hline lap. CCE bis 6 / 2009 & 332 & & 6,35 & 1,22 & 2,56 \\
\hline lap. CCE ab 7/2009 & 25 & 5 & 4,68 & 0,84 & 2,43 \\
\hline lap. HP bis 6/2009 & 345 & & 4,46 & 0,76 & 2,31 \\
\hline lap. HP ab 7 / 2009 & 26 & 10 & 3,96 & 0,35 & 2,47 \\
\hline kein PB & 15 & & 5,53 & 1,33 & \\
\hline
\end{tabular}

Tab. 1 Darstellung der Gesamtoperationen, der Liegedauer, der präop. Tage und des Schmerzempfindens in den verschiedenen Gruppen der laparoskopischen Cholezystektomie (CCE) und Hernioplastik (HP). 
konnte jedoch die Wartezeit bei der Erstanlage der Behandlungspfade auf $20 \mathrm{~s}$ und bei jedem weiteren Aufruf auf $5 \mathrm{~s}$ verringert werden, sodass der Pfad jetzt auch während der Visite bettseitig durchgeführt wird.

Überraschend, trotz aller dargestellten Probleme, war es daher für uns, dass in der Allgemeinchirurgie nach der Umstellung der etablierten papiergebundenen Pfade auf IT-gestützte Behandlungspfade sich die Rate der nicht nach Pfad behandelten Patienten oder Pfadabbrüche im Rahmen der Voruntersuchungen bewegte. Da die reinen Zahlenwerte etwas erhöht anmuten, möchten wir anmerken, dass als Pfadabbruch jede Verlängerung der präoperativen Phase und jede Verlängerung der Verweildauer z.B. durch eine internistische Begleiterkrankung, Komplikationsdiagnosen, den Wechsel des Operationsverfahrens (z.B. offene Cholezystektomie statt laparoskopischen Verfahren) gewertet wurde. Die Ursachen der Pfadabbrüche liegen demzufolge nicht ausschließlich in der Komplexität der Behandlungspfade. Ebenfalls positiv ist der Umstand zu werten, dass sich auch im subjektiven Schmerzempfinden und in der Gesamtliegedauer der Patienten keine Veränderung ergeben hatte. So kann hier postuliert werden, dass eine Umstellung zur IT-gestützten Behandlungspfadführung nicht zu einer Veränderung der Prozess- und Ergebnisqualität führt. Der Umstand der Verringerung der präoperativen Verweildauer ist unserer Meinung nach insbesondere der DRG-Einführung im Untersuchungsintervall geschuldet. Da unsere Betrachtung bereits 1.1.2000 beginnt, schließt sie den Zeitraum der DRG-Einführung ein und diese hat insbesondere bei Elektiveingriffen zu einer Verlagerung der präoperativen Diagnostik in den prästationären Bereich geführt $[18,19]$.

Somit liegen die Vorteile des IT-gestützten Behandlungspfades als Weiterentwicklung bestehender papiergebundener Pfade nicht in der weiteren Qualitätsverbesserung, sondern vielmehr in der Arbeitserleichterung durch den Wegfall der Parallelität einer Papier- und Elektronikkurve, sodass nunmehr Anordnungen und Dokumentationen nicht mehr doppelt durchgeführt werden mussten. Weiterhin können IT-gestützte Behandlungspfade zügig an die sich im permanenten Fluss befindlichen klinikinternen Gegebenheiten angepasst werden. So haben wir die Umstellung der ärztlichen Anamneseerhebung von der Papierform in ein elektronisches Formular zeitnah in den Pfad integriert. Gleiches betrifft die Integration der Operationsanmeldung und Erstellung eines standardisierten, für den jeweiligen Patienten angeglichenen Arztbriefes. Darüber hinaus tragen Behandlungspfade aufgrund der verpflichtenden Eingabe zur lückenlosen Dokumentation bei. Dies konnten wir am Beispiel der postoperativen Schmerzen zeigen, welche zu den häufigsten im klinischen Alltag auftretenden Akutschmerzen gehören und zum Anstieg postoperativer Komplikationen führen, deren Folgen ein verlängerter Krankenhausaufenthalt, eine Letalitätserhöhung und ein Anstieg der Behandlungskosten sind. Unser Ziel war es, durch die regelmäßige elektronische Datenerfassung des subjektiven Schmerzempfindens allgemeinchirurgischer Patienten die Qualität der postoperativen Schmerztherapie zu verbessern, denn eine Schmerzerfassung ist eine unverzichtbare Voraussetzung für die individuell angepasste Therapie [2022]. Leider konnten wir eine Verbesserung der postoperativen Schmerztherapie statistisch nicht für beide Gruppen nachweisen, aber die Frequenz der Schmerzerfassung hatte sich auf über $2 \mathrm{Er}$ fassungen pro Tag erhöht, sodass wir von einer engmaschigeren Kontrolle unserer operierten Patienten im Sinne der Schmerzüberwachung ausgehen können.

\section{Zusammenfassung}

$\nabla$

Klinische Behandlungspfade sind der berufsgruppenübergreifende Konsens einer Behandlung zur Steuerung und Dokumentation des Behandlungsprozesses unter optimaler Ressourcennutzung und Festlegung der Durchführungs- und Ergebnisverantwortlichkeit. Ein IT-gestützter klinischer Behandlungspfad ist als Routinearbeitsinstrument im Krankenhausinformationssystem (KIS) als kontinuierliche Fortsetzung klinischer papiergebundener Behandlungspfade einsetzbar und kann für einzelne operative Prozeduren als umfassendes klinisch-administratives Steuerungsinstrument angewandt werden.

Dabei kann eine Ökonomisierung der Behandlungsabläufe erreicht werden. Die bereits durch die etablierten Papier-Pfade erreichte Prozess- und Ergebnisqualität wird durch die IT-gestützte Pfadführung nicht beeinflusst. Wir erreichen jedoch eine automatisierte, lückenlose und rechtlich sichere Dokumentation im Rahmen der elektronischen Fieberkurve sowie eine Arbeitserleichterung durch den Wegfall von Doppeleintragungen oder Anforderungen in Papierform und IT-gestützt. Darüber hinaus lassen sich neben der Verbesserung von Transparenz und Behandlungsabläufen, auch Ausbildungseffekte für neue Mitarbeiter erzielen. Dadurch ist eine schnellere Einarbeitung der Mitarbeiter in Kliniken mit einer hohen Fluktuation möglich. Für diese neuen Mitarbeiter bieten Behandlungspfade zunächst einen „roten Faden“, um sich in den Stations- und Klinikalltag einzuarbeiten. IT-gestützte Behandlungspfade können auch zur Evaluierung qualitativer und ökonomischer Aspekte und somit sehr zeitnah zur Verbesserung der Behandlungsabläufe genutzt werden.

Aus diesen Gründen sollten Krankenhäuser, die bereits eine umfassende elektronische Behandlungsführung besitzen, ihre Behandlungsabläufe mit IT-gestützten Behandlungspfaden optimieren.

\section{Interessenkonflikt: Nein.}

\section{Literatur}

1 Cheney J, Barber S, Altamirano L et al. A clinical pathway for bronchiolitis is effective in reducing readmission rates. J Pediatr 2005; 147: 622626

2 Choong PF, Langford AK, Dowsey MM et al. Clinical pathway for fractured neck of femur: a prospective, controlled study. Med J Aust 2000; 172: 423-426

3 Dowsey MM, Kilgour ML, Santamaria NM et al. Clinical pathways in hip and knee arthroplasty: a prospective randomised controlled study. Med J Aust 1999; 170: 59-62

4 Hauck LD, Adler LM, Mulla ZD. Clinical pathway care improves outcomes among patients hospitalized for community-acquired pneumonia. Ann Epidemiol 2004; 14: 669-675

5 Pearson SD, Goulart-Fisher D, Lee TH. Critical pathways as a strategy for improving care: problems and potential. Ann Intern Med 1995; 123 : 941-948

6 Schuld J, Richter S, Folz J et al. Einfluss IT-gestützter Behandlungspfade auf die Patientenzufriedenheit an einer chirurgischen Universitätsklinik. Dtsch Med Wochenschr 2008; 133: 1235-1239

7 Vuori H. Patient satisfaction - does it matter? Qual Assur Health Care 1991; 3: 183-189

8 Ransom SB, Studdert DM, Dombrowski MP et al. Reduced medicolegal risk by compliance with obstetric clinical pathways: a case-control study. Obstet Gynecol 2003; 101: 751-755

9 Ronellenfitsch U, Rössner E, Jakob J et al. Clinical Pathways in surgery should we introduce them into clinical routine? A review article. Langenbecks Arch Surg 2008; 393: 449-457

10 Roeder N, Hindle D, Loskamp $N$ et al. Frischer Wind mit klinischen Behandlungspfaden. Das Krankenhaus 2003; 1: 20-27 
11 Schwarzbach $M$, Ronellenfitsch $U$. Klinikpfade in der Chirurgie. Ein Instrument für den Routinebetrieb? Dtsch Arztebl 2008; 105: A2512A2516

12 Ronellenfitsch $U$, Schwarzbach M. Klinisches Prozessmanagement - Klinische Pfade in der Chirurgie: Evidenz und Potenzial. Zentralbl Chir 2010; 135: 99-101

13 Markey DW, McGowan J, Hanks JB. The effect of clinical pathway implementation on total hospital costs for thyroidectomy and parathyroidectomy patients. Am Surg 2000; 66: 533-538

14 Pritts TA, Nussbaum MS, Flesch LV et al. Implementation of a clinical pathway decreases length of stay and cost for bowel resection. Ann Surg 1999; 230: 728-733

15 Stephen AE, Berger DL. Shortened length of stay and hospital cost reduction with implementation of an accelerated clinical care pathway after elective colon resection. Surgery 2003; 133: 277-282

16 Uchiyama K, Takifuji K, Tani M et al. Effectiveness of the clinical pathway to decrease length of stay and cost for laparoscopic surgery. Surg Endosc 2002; 16: 1594-1597
17 Schilling $M K$, Richter S, Jacob $P$ et al. Klinische Behandlungspfade Erste Ergebnisse des systematischen IT-gestützten Einsatzes an einer chirurgischen Universitätsklinik. Dtsch Med Wochenschr 2006; 131: 962-967

18 Cushing KA, Stratta RJ. Design, development and implementation of a critical pathway in simultaneous pancreas-kidney transplant recipients. J Transpl Coord 1997; 7: 164-172

19 Napolitano LM. Standardization of perioperative management: clinical pathways. Surg Clin North Am 2005; 85: 1321-1327

20 Neugebauer E, Sauerland S, Keck Vet al. Leitlinien Akutschmerztherapie und ihre Umsetzung in der Chirurgie. Chirurg 2003; 74: 235-238

21 Stamer U, Mpasios N, Stüber F et al. Postoperative Schmerztherapie in Deutschland. Ergebnisse einer Umfrage. Anaesthesist 2002; 51: 248257

22 Vereinbarung zur Organisation der postoperativen Schmerztherapie des Berufsverbandes Deutscher Anästhesisten und des Berufsverbandes Deutscher Chirurgen. Anästhesiol Intensivmed 1993; 34: 2832 